\title{
Ice-sheet elevations from across-track processing of airborne interferometric radar altimetry
}

\author{
R. L. Hawley, ${ }^{1,2}$ A. Shepherd, ${ }^{3}$ R. Cullen, ${ }^{4}$ V. Helm, ${ }^{5}$ and D. J. Wingham ${ }^{6}$ \\ Received 5 August 2009; revised 24 September 2009; accepted 16 October 2009; published 19 November 2009.
}

[1] Interferometric Radar Altimeters (IRA's) use dual receive antennas to overcome one of the spatial limitations of pulse-limited altimeters. In a conventional IRA measurement, the range and across-track direction of a scatterer are determined using the phase difference between the antennas. We demonstrate a method of determining multiple elevation points across a swath orthogonal to the instrument ground track in regions of steep terrain, such as ice-sheet margins. We use data from an airborne IRA (a prototype of the CryoSat-2 instrument), and compare the results to simultaneous Airborne Laser Scanner (ALS) observations. This application results in a 75-fold increase in measurement density compared to conventional radar altimetry. Along a $\sim 2.5 \mathrm{~km}$ ground track, the RMS departure between the IRA- and ALS-derived measurements was $1.67 \mathrm{~m}$. Based on our result, although our approach is limited to areas of relatively steep slope, a 25 - to 75 -fold increase in elevation measurements could be achieved in coastal regions of Antarctica and Greenland with similar processing of CryoSat-2 data. Citation: Hawley, R. L., A. Shepherd, R. Cullen, V. Helm, and D. J. Wingham (2009), Icesheet elevations from across-track processing of airborne interferometric radar altimetry, Geophys. Res. Lett., 36, L22501, doi:10.1029/2009GL040416.

\section{Introduction}

[2] Understanding the state of the world's ice masses is important to scientists, policymakers, and society. Although the large ice sheets of Antarctica and Greenland contain the vast majority of ice on Earth today, the world's $10^{5}$ small glaciers and ice caps are expected to provide the greatest cryospheric component of 21 st century sea level rise [Lemke et al., 2007]. These small ice masses include steep topography which has limited our ability to quantify changes in their volume using radar altimetry. In addition, the sectors of the Antarctic and Greenland ice sheets exhibiting the greatest changes today are at their margins, where slopes are similarly problematic. The CryoSat-2 interferometric radar altimeter (IRA) is designed to overcome some of the limitations of pulse-limited altimeters [e.g., Partington,

\footnotetext{
${ }^{1}$ Department of Earth Sciences, Dartmouth College, Hanover, New Hampshire, USA.

${ }^{2}$ Scott Polar Research Institute, Cambridge University, Cambridge, UK.

${ }^{3}$ School of Geosciences, University of Edinburgh, Edinburgh, UK.

${ }^{4}$ ESTEC, European Space Agency, Noordwijk, Netherlands.

${ }^{5}$ Alfred Wegener Institute for Polar and Marine Research, Bremerhaven, Germany.

${ }^{6}$ Centre for Polar Observation and Modelling, University College London, London, UK.
}

Copyright 2009 by the American Geophysical Union. 0094-8276/09/2009GL040416
1998] and survey ice elevation changes with sufficient spatial and temporal resolution to fill gaps in our knowledge of such regions [Wingham et al., 2005].

\section{Background}

[3] In sloping regions, the first echo returned to the receiver will be from the off-nadir Point Of Closest Approach (POCA). A classical pulse-limited altimeter cannot determine the location of the POCA without additional slope information. An IRA uses dual receive antennas to determine the across-track location of the POCA, and the angle subtended to the scattering location can be determined using the phase difference between echoes received at the two antennas.

[4] In the normal operation of an IRA over a flat or slightly-sloping surface, the echo from the POCA (point 0 in Figure 1d) will be from a location within the antenna beamwidth. Subsequent echoes will then simultaneously arrive from both sides (across-track) of the POCA (Figures 1d, top and 1d, middle), and the phase-difference signal will be indistinct. In this case, the correct identification of the POCA using interferometric phase ("conventional processing") is possible, but the across-track processing scheme presented here would be foiled. If, however, the surface slope is large enough that the POCA is on the edge of or outside the antenna beam (i.e., when the surface slope is greater than half of the antenna's angular beamwidth, or about $1.25^{\circ}$ for ASIRAS), the echoes from outside the beam will be sufficiently attenuated that the recorded phase signal is from only one side of the POCA, and can thus be used to determine the elevation and position of successive points in the acrosstrack direction (Figure 1d, bottom). Note that a similar geometry could be achieved over flat terrain by angling the antenna.

[5] Here, we extend the interferometric altimetry technique of Jensen [1999]. An echo from an angle $\theta$ off-nadir will be received by one antenna slightly before the other, as it travels a slightly shorter path. This path length difference is equal to $b \sin (\theta)$ where $b$ is the length of the antenna baseline. The phase difference between the signals reaching the two antennas will be $(2 \pi / \lambda) b \sin (\theta)$ where $\lambda$ is the wavelength of the carrier frequency. The relationship between the angle $\theta$ (from the antenna baseline to the target) and the measured phase difference $\phi$ between the two antennas is given by

$$
\phi=\kappa b \sin (\theta) \approx \kappa b \theta
$$

where $\kappa=2 \pi / \lambda$ is the wavenumber of the carrier frequency.

[6] We extend this relationship to include the echoes acquired from the remainder of the coherent backscattered 

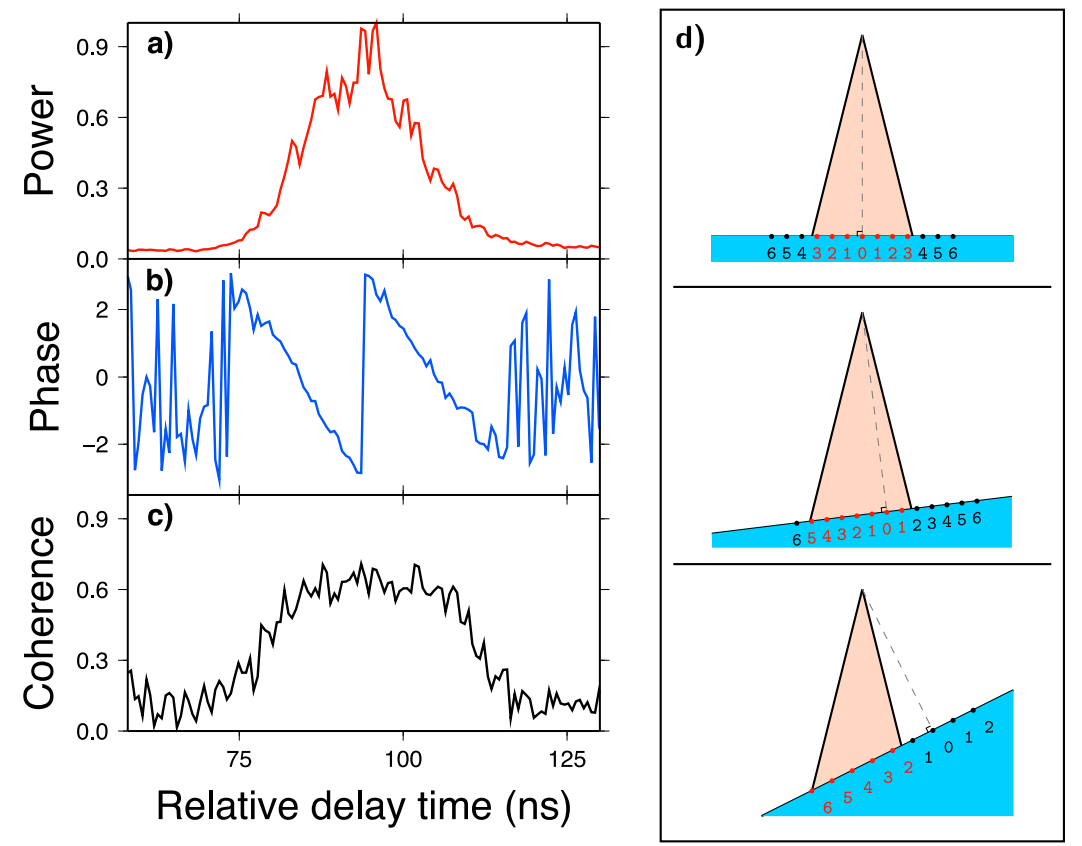

Figure 1. An example record from our ASIRAS Interferometric Radar Altimeter data. (a) Normalized power, (b) interferometric phase difference (radians), (c) coherence. Clearly evident is the phase "ramp" as the point of intersection between the radar wave and the ground sweeps across the ground track. (d) Geometry of the measurement over an ice sheet at 3 different slopes. The antenna beamwidth is indicated by the shaded red triangle. Altimeter returns at successive times arrive at the antenna from the numbered points, with point 0 being the Point of Closest Approach (POCA). (top and middle) Successive returns come from both sides within the beam pattern. In the second case, at moderate slope, the POCA is shifted to the right. In this case the altimeter operating in interferometric mode can still correctly identify the POCA. (bottom) Illustrated is the geometry required for our across-track processing. Here, the POCA is outside the antenna beam pattern, and so returns from upslope of the POCA are attenuated and do not contribute to the recorded phase signal. The remaining points $2-6$ have a simple phase-distance relationship, and can be processed into multiple across-track elevations.

echo waveform. At each location sampled within the waveform, the phase measurement provides the look angle and the time delay provides the range to the scattering surface. It is then a straightforward geometric translation, knowing the aircraft attitude and thus the attitude of the antenna baseline, to determine the elevation of the ground location from which echoes are returned.

\section{Data and Methods}

\subsection{Location}

[7] We use data collected during the CryoSat Validation Experiment (CryoVEx) 2004 spring campaign, over the Austfonna ice cap in Svalbard. At this low-elevation site near the equilibrium line [Pinglot et al., 2001], the radar return is dominated by surface scattering, and penetration of the radar pulse [e.g., Hawley et al., 2006] is minimal. Under such circumstances, the interferometric phase is directly related to off-nadir surface scattering.

\subsection{ASIRAS}

[8] The Airborne Synthetic aperture Interferometric Radar Altimetry System (ASIRAS) [Lentz et al., 2002] is an IRA, designed to demonstrate the concept of a satellite based system for the CryoSat mission [Wingham et al., 2005]. The radar is a phase sensitive Ku band altimeter with a high pulse repetition frequency, allowing along-track synthetic aperture beam-forming. Beam-forming is per- formed using a method [Wingham et al., 2004] similar to the delay-doppler concept [Raney, 1998; Raney and Leuschen, 2004] to optimize along-track resolution by taking multiple looks in the along-track direction. The carrier frequency of the radar is $13.5 \mathrm{GHz}$ and the bandwidth is $1 \mathrm{GHz}$. The half-power beamwidth before processing is approximately 2.5 degrees in the across-track direction and 10 degrees in the along-track direction. Aircraft position and attitude are measured with a Differential Global Positioning System (DGPS) and an Inertial Navigation System (INS), respectively.

[9] We adjusted the raw, un-calibrated radar data to compensate for the effects of the delay-doppler slant-range geometry [Wingham et al., 2004]. Adjusted waveforms scattered from the same along-track location were averaged (multi-looked) and then geo-located using information from the DGPS and the INS. Figure 1 shows an example record from our test site. A phase "ramp", which results from the changing angle between the interferometer baseline and the point from which the signal is returned, is clearly evident. In addition, a discontinuity can be seen where the interferometric phase exceeds $\pm 2 \pi$ and thus wraps. In this study, the amplitude (power) and phase of radar echoes are processed to form a single-look complex image, and the phase is subsequently filtered and unwrapped to remove ambiguities [Werner et al., 2002].

[10] The unwrapped phase signal forms the basis of our across-track elevation measurements. Each echo sample 

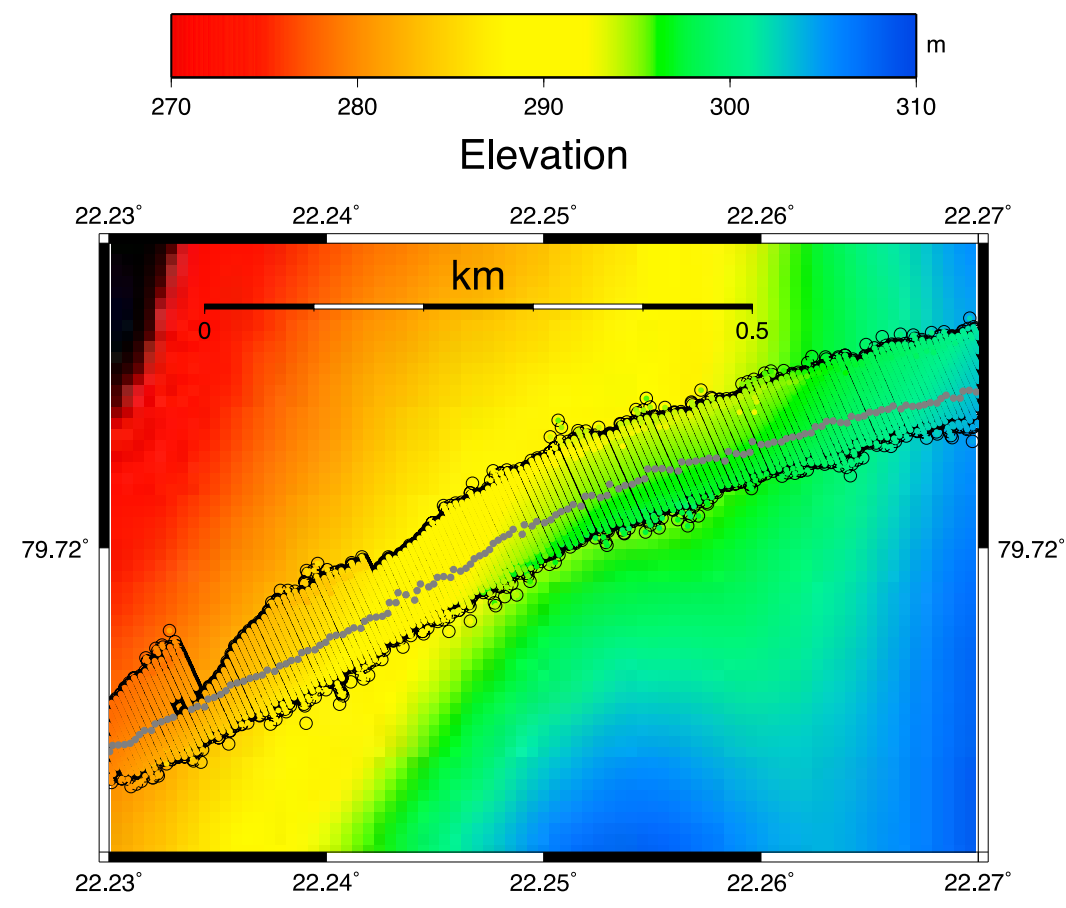

Figure 2. A section of our across-track processing result. Gridded elevations from the Airborne Laser Scanner form the background. Each across-track-processed (angle, distance) point is plotted as a filled circle with the same elevation colormap. Gray circles indicate the positions of elevation points obtained with conventional processing; though the actual Point Of Closest Approach is at the edge of our swath, imperfect retracking of the echoes in conventional processing results in a retracked POCA near the center of our swath. Clearly we successfully calculate across-track positions and elevations from the altimetric phase measurement.

originates from a discrete angle with respect to the interferometer baseline, and the time of each phase measurement indicates the distance from the antenna phase center to the scattering point. For each range bin we compute the vector between the antenna phase center and the scattering point. This vector is in an inertial reference frame tied to the aircraft. We then use the DGPS and INS data to determine the aircraft position and attitude, and combine the angles of pitch, roll, and aircraft heading in a co-ordinate transformation to determine the geodetic (WGS-84) coordinates of the ground point. Figure 2 shows an example of elevation measurements derived in this way.

\subsection{Airborne Laser Scanner}

[11] We use data from a commercial Airborne Laser Scanner (ALS; Rigel LMS-Q280) mounted on the same aircraft platform to validate the IRA elevation measurements. The LMS-Q280 uses a $1064 \mathrm{~nm}$ laser and a scanning mirror to collect measurements at $\sim 2-3 \mathrm{~m}$ ground spacing. Ice surface elevations from the ALS have proven to be repeatable to within $\pm 10 \mathrm{~cm}$ (V. Helm et al., unpublished field report, 2006), making the ALS data ideal for validation. Data collected from runway overflights revealed a constant offset of $\sim 0.8 \mathrm{~m}$ betwen ALS and retracked ASIRAS elevations. However, this is roughly equal to the distance between first energy return and the retracked point on the waveform. Because our processing uses the full waveform and no retracking, we compare elevations using an offset of zero.

[12] We gridded the ALS elevations and calculated an interpolated ALS elevation for each ASIRAS-resolved ground point. The number and accuracy of the ASIRAS elevation measurements are each dependent on the coherence threshold at which the interferometric data are unwrapped (Table 1). On average, an $\sim 85 \mathrm{~m}$ swath of elevation measurements was obtained with a root-mean square departure of $\sim 1.6 \mathrm{~m}$ with respect to the ALS observations.

\section{Discussion}

\subsection{Performance of the Algorithm}

[13] The two-dimensional grid of elevation measurements retrieved using this interferometric processing algorithm provide vastly improved coverage compared to conventional processing, which would have otherwise provided only a one-dimensional line of elevation points near to the ground track (Figure 2). At our experiment site, the elevation ranged from $\sim 250 \mathrm{~m}$ to $\sim 330 \mathrm{~m}$ along track, with

Table 1. Elevation Comparison Between Across-Track Processed ASIRAS Radar and Gridded ALS Data ${ }^{a}$

\begin{tabular}{ccc}
\hline Coherence Band & RMS $(\mathrm{m})$ & Number of points \\
\hline $0.4-0.5$ & 2.08 & 3109 \\
$0.5-0.6$ & 1.86 & 4812 \\
$0.6-0.7$ & 1.63 & 5473 \\
$0.7-0.8$ & 1.36 & 5967 \\
$0.8-0.9$ & 1.39 & 6023 \\
$0.9-1.0$ & 1.78 & 8443 \\
All points & 1.67 & 33828 \\
POCA only & 1.33 & 448 \\
\hline
\end{tabular}

${ }^{\mathrm{a}} \mathrm{RMS}$ is the root-mean-squared difference between the ASIRAS and ALS data. The last line shows the results if no across-track processing is applied, as would be the case for a conventional level-2 elevation product. 


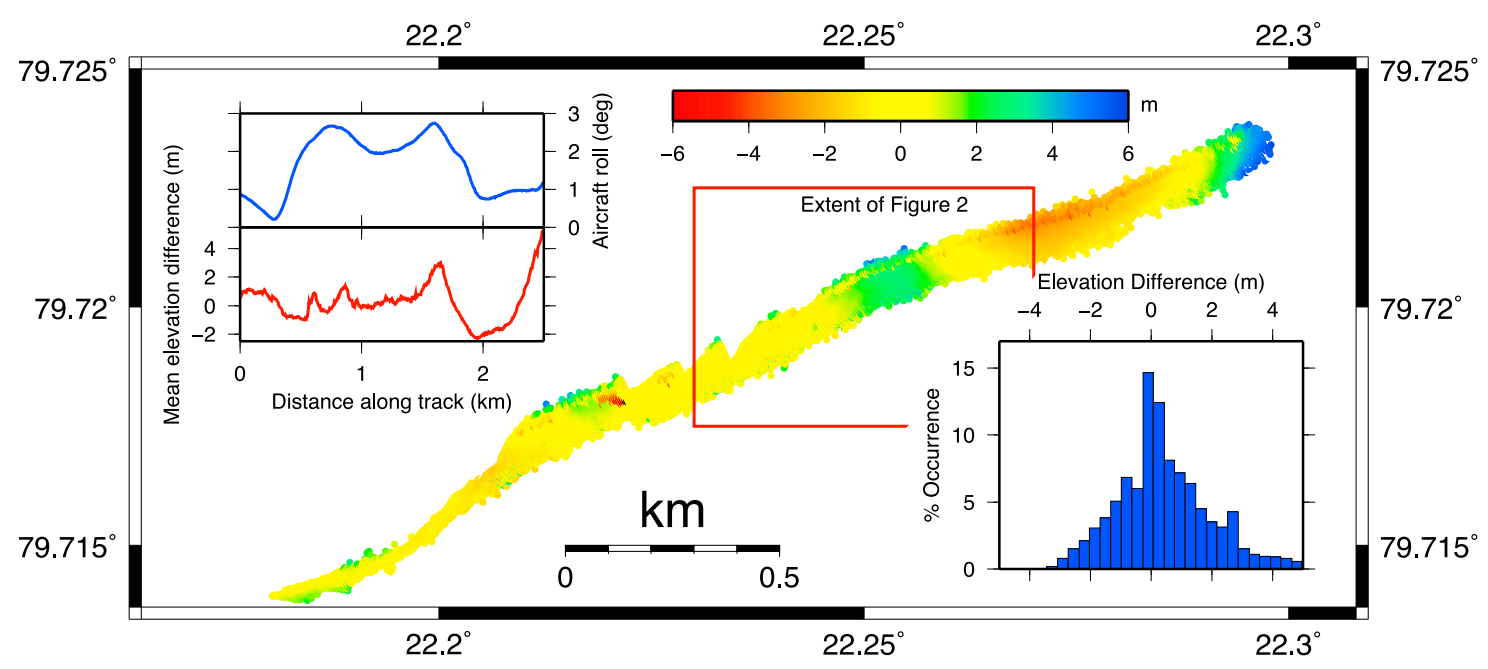

Figure 3. Elevation differences between our across-track processed radar result and ALS data. The inset at lower right shows a histogram of the values from the 33,828 points plotted. The high-difference areas show a spatial pattern; this may be due to errors associated with rapid changes in aircraft attitude. The inset at upper left shows the mean elevation difference averaged along track, and the aircraft roll angle. The red box outlines the area illustrated in Figure 2.

across-track elevation differences of 1 to 10 meters. The effective surface slope (angle between the surface and the interferometric baseline of the antenna, which varies with the attitude of the aircraft) varied from approximately 2.5 to 5.5 degrees.

[14] Over our $\sim 2.5 \mathrm{~km}$-length flightline, our acrosstrack processing resulted in 33,828 individual elevation point measurements (Table 1), a 75 -fold increase over the 448 points that result from conventional "POCA" processing. The departure from the ALS elevations is slightly higher for our across-track processing result, but this is partly due to the use of lower-coherence points. Using only the subset of points with coherence between 0.7 and 0.9 , the resulting RMS difference is $1.37 \mathrm{~m}$ (close to the "POCA" processing RMS of $1.33 \mathrm{~m}$ ), and the number of points is 11,990 , a 26-fold increase in point density over conventional processing. It should be noted here that for any given waveform, the additional points share any elevation bias associated with that waveform.

[15] As shown in Figure 3, the spatial pattern of difference between ASIRAS- and ALS-derived elevations is more pronounced along-track than across-track. This may be due to errors associated with rapid changes in aircraft attitude. Many of the higher-difference regions were recorded while the aircraft roll or rate-of-roll was changing rapidly (Figure 3, inset). While no definitive relationship was found between aircraft attitude and elevation difference, changes in the antenna baseline would affect the received phase difference, and a finite response time by the INS could induce errors during rapid attitude changes.

[16] The phase-unwrapping algorithm requires a point of known "absolute" phase from which to unwrap. If this point is chosen at a location where the phase has already wrapped, a $2 \pi$ ambiguity could be introduced. For ASIRAS, the effect of the entire phase result being shifted by $2 \pi$ is to change the across-track angle by $\sim 1.7$ degrees. Since the across-track distance would change while the range would remain the same, a slope-dependent error in retrieved elevation would be introduced. With our geometry, a $2 \pi$ error in phase results in elevation errors $> \pm 2$ meters.
Because our elevations are much closer to the ALS result than this, we are confident that our algorithm has successfully captured the true phase signal.

\subsection{Application to Space-Borne Radar Altimeters}

[17] We investigated the possibility of deriving similar elevation data from observations of the planned CryoSat-2's Synthetic aperture Interferometric Radar Altimeter (SIRAL). By increasing the number of elevation measurements available one potentially achieves greater spatial coverage of steep ice-covered terrain, such as the margins of Antarctica and Greenland, which are currently omitted from altimeter surveys of ice volume trends [Wingham et al., 2006].

[18] The swath width achievable with SIRAL differs from that of ASIRAS due to differences between key parameters of the two systems. In particular, ASIRAS was flown for our study at approximately 1140 meters above the surface, and recorded 256 samples at a slant-range spacing of $0.086 \mathrm{~m}$, with an antenna pattern half-power beamwidth of 2.5 degrees across-track. Flying at an altitude of $\sim 717 \mathrm{~km}$, SIRAL will collect 512 samples per waveform, at a nominal slant-range spacing of $0.47 \mathrm{~m}$. The across-track beamwidth is 1.2 degrees. Under perfect conditions for across-track processing, this would correspond to an across-track range of over $18 \mathrm{~km}$. If phase coherence is preserved across-track over $\sim 1 / 3$ of each waveform in SIRAL data as we have observed in ASIRAS measurements, elevation data at swath widths of up to $6 \mathrm{~km}$ are to be expected. In practice, however, as the interferometer look-angle approaches the theoretical limit for across-track processing, which is less than the antenna half power beamwidth, or 1.2 degrees for SIRAL, the phase "ramp" steepens, and the wrapped signal is resolved by fewer and fewer samples. In these areas phase unwrapping is expected to become problematic and swath widths will become smaller until only a single point can be resolved.

[19] In addition to improved spatial coverage, acrosstrack processing can provide improved temporal coverage. For change detection, crossover analysis is commonly used 
[e.g., Wingham et al., 1998]. By increasing the number of elevations measured across-track, we increase the number of crossovers for any given orbit as well. Thus, changes can be tracked over shorter time-scales and with greater accuracy than with conventional radar altimeters [e.g., Wingham et al., 1998].

\section{Conclusions}

[20] We have demonstrated the ability to derive ice surface elevation using interferometric synthetic aperture radar altimetry across a wide swath orthogonal to the instrument ground track in a region of sloping terrain. When applied to IRA data recorded from an aircraft, the method provides a 75 fold increase in the number of elevation measurements in a region of favorable (high) slope, with 33,828 elevation points at an RMS difference of $1.67 \mathrm{~m}$, compared with 448 conventionally-processed elevation points with an RMS difference of $1.33 \mathrm{~m}$. Successful application of similar processing to data from the upcoming CryoSat-2 satellite-based IRA would improve observations of volume trends in regions of the cryosphere that are currently omitted from pulse-limited altimeter surveys, such as the steep margins of Antarctica and Greenland and smaller ice caps.

[21] Acknowledgments. This work is supported by the UK Natural Environmental Research Council and the European Space Agency. We thank Constantin Mavrocordatos and everyone involved in the development of the ASIRAS instrument at Radar Systematik (RST) and ESA. We thank all team members involved in the CryoVEx 2004 campaign, particularly the air crew who flew the radar system, including Tobias Boebel of Optimare and Harald Lenz of RST.

\section{References}

Hawley, R. L., E. M. Morris, R. Cullen, U. Nixdorf, A. P. Shepherd, and D. J. Wingham (2006), ASIRAS airborne radar resolves internal annual layers in the dry-snow zone of Greenland, Geophys. Res. Lett., 33, L04502, doi:10.1029/2005GL025147.

Jensen, J. R. (1999), Angle measurement with a Phase-Monopulse radar altimeter, IEEE Trans. Antennas Propag., 47, 715-724.
Lemke, P., et al. (2007), Observations: Changes in snow, ice and frozen ground, Climate Change 2007: The Physical Science Basis. Contribution of Working Group I to the Fourth Assessment Report of the Intergovernmental Panel on Climate Change, edited by S. Solomon et al., pp. 337383, Cambridge Univ. Press, Cambridge, U. K.

Lentz, H., H.-M. Braun, M. Younis, C. Fletcher, W. Wiesbeck, and C. Mavrocordatos (2002), Concept and realization of an airborne SAR Interferometric Radar Altimeter System (ASIRAS), in Geoscience and Remote Sensing Symposium, IGARSS '02 Proceedings, vol. 6, pp. 30993101, Inst. of Electr. and Electron. Eng., New York.

Partington, K. C. (1998), Antenna beamwidth and antenna pointing-induced errors in topographic monitoring from satellite radar altimeters, IEEE Trans. Geosci. Remote Sens., 36, 312-316.

Pinglot, J. F., J. O. Hagen, K. Melvold, T. Eiken, and C. Vincent (2001), A mean net accumulation pattern derived from radioactive layers and radar soundings on Austfonna, Nordaustlandet, Svalbard, J. Glaciol., 47, $555-566$.

Raney, R. K. (1998), The delay/Doppler radar altimeter, IEEE Trans. Geosci. Remote Sens., 36, 1578-1588.

Raney, R. K., and C. J. Leuschen (2004), Simultaneous laser and radar altimeter measurements over land and sea ice, in Geoscience and Remote Sensing Symposium: IGARSS '04 Proceedings, vol. 1, p. 678, Inst. of Electr. and Electron. Eng., New York.

Werner, C., U. Wegmuller, T. Strozzi, and A. Weisman (2002), Gamma SAR and interferometric processing software, paper presented at the ENVISAT/ERS Symposium, Eur. Space Agency, Gothenburg, Sweden.

Wingham, D. J., A. J. Ridout, R. Scharroo, R. J. Arthern, and C. K. Schum (1998), Antarctic elevation change from 1992 to 1996, Science, 282, $456-458$.

Wingham, D. J., L. Phalippou, C. Mavrocordatos, and D. Wallis (2004), The mean echo and echo cross product from a beamforming interferometric altimeter and their application to elevation measurement, IEEE Trans. Geosci. Remote Sens., 42, 2305-2323.

Wingham, D. J., et al. (2005), CryoSat: A mission to determine the fluctuations in Earth's land and marine ice fields, Adv. Space Res., 37, 841-871.

Wingham, D. J., A. Shepherd, A. Muir, and G. J. Marshall (2006), Mass balance of the Antarctic ice sheet, Philos. Trans. R. Soc., Ser. A, 364, $1627-1635$.

R. Cullen, ESTEC, European Space Agency, Keplerlaan 1, Postbus 299, NL-2200 AG Noordwijk, Netherlands.

R. L. Hawley, Department of Earth Sciences, Dartmouth College, HB6105 Fairchild Hall, Hanover, NH 03755, USA. (robert.l.hawley@ dartmouth.edu)

V. Helm, Alfred Wegener Institute for Polar and Marine Research, Am Alten Hafen 26, D-27568 Bremerhaven, Germany.

A. Shepherd, School of Geosciences, University of Edinburgh, Edinburgh EH8 9XP, UK.

D. J. Wingham, Centre for Polar Observation and Modelling, University College London, Pearson Bldg., Gower St., London WC1E 6BT, UK. 